\title{
Development of Mobile Indoor Positioning System Application Using Android and Bluetooth Low Energy with Trilateration Method
}

\author{
Agustinus Noertjahyana ${ }^{1}$, Ignatius Alex Wijayanto ${ }^{2}$, Justinus Andjarwirawan ${ }^{3}$ \\ Informatic Engineering, Faculty of Industrial Technology, Petra Christian University \\ Jl. Siwalankerto 121-131 Surabaya 60236 \\ agust@petra.ac.id ${ }^{1}$, isax.clairene@google.com², justin@petra.ac.id ${ }^{3}$
}

\begin{abstract}
The development of information technology and the concept of smart city began to grow. Information technology needed by the public that is looking for information position and location of destination in the building (indoor positionting). Indoor positioning using $\mathrm{WiFi}$ has limits on location placement. To overcome these shortcomings are used sensor beacon bluetooth low energy with the advantages of having low power consumption and relatively small dimensions that can be placed in various places that are difficult to reach by WiFi.
\end{abstract}

Indoor Positioning System (IPS) is a system for knowing the position of objects or people in a room by using radio waves, magnetic fields, or other sensors obtained by mobile devices. The indoor positioning method is divided into deterministic and probabilistic. Deterministic can determine the position faster by using measurement techniques such as Trilateration and Triangulation. Trilateration is a method for determining location with known three location information and device distance to each access point. This research used Trilateration method with measurement technique based on RSSI value.

Based on the results of the test, RSSI of the beacon is strongly affected by objects that have thickness and density. In line of sight conditions, Android devices are able to receive signals properly and determine the location of the device using trilateration with quite accurate.

Keywords: Indoor Positioning System, Trilateration, Bluetooth Low Energy, Android.

\section{INTRODUCTION}

In this era of technology, the concept of smart city began to develop, where information technology is integrated into a city. One of the information technology required by the community is the indoor map, where the community is having difficulty to know their location dan the destination in the building that have never been visited or have many rooms. This building can be in public places like malls, stations, airports, hospitals, and universities.

Methods in indoor positioning are divided into two parts: deterministic and probabilistic. Deterministic methods can provide faster position estimation using classical measurement techniques such as Trilateration and Triangulation. This technique uses a simple signal strengh map where at each location has a number of objects or services that have a signal strength value. The probabilistic method works by estimating the location by accurately calculating the location of the object with the current calculation. This method requires more information than deterministic methods so that this method has more accurate location detection. This method can not work on devices with small capacities such as sensors because this method requires a lot of computing other than that this method takes a long time to give the results of calculations.

To answer the problems, in this research using Trilateration method to determine the position of the device. Measurement techniques that can be done on this method are Received Signal Strength (RSS), Time of Arrival (ToA), and Time Difference of Arrival (TDoA). This method requires three mobile device positions to each transmitter to get optimal position.

Indoor Positioning System (IPS) is a system for knowing the position of objects or people in a room using radio waves, magnetic fields, or other sensors obtained by mobile devices [1]. IPS can utilize Global Positioning System (GPS). However, GPS has a disadvantage that is the dependence on the location around which has access point and line-of-sight from satellite. WiFi is the access point most commonly found in public places, though WiFi has some disadvantages that requires power consumption that comes from the electrical connection so that it has limitations in the placement of this device. To resolve this problem, IPS can utilize a beacon bluetooth low energy (BLE), which is capable of emitting signals with low power consumption coming from the battery. Beacon BLE also has a small dimension so that this device can be placed in various places.

So the advantages of BLE beacons are used for this research, in this study made a mobile application that utilizes indoor positioning system technology using Android and low-energy bluetooth. This app is created to provide indoor maps services by utilizing BLE to help users find in-building user positions with more accurate accuracy than using GPS.

\section{THEORY}

\section{A. Indoor Positioning System (IPS)}

Indoor Positioning System (IPS) is a system for knowing the position of objects or people in a room by using radio waves, magnetic fields, or other sensors obtained by mobile devices [1]. IPS by using radio waves have advantages over other sensors because the technology on mobile devices capable of processing radio waves that exist around the user. Technology that can emit radio waves and commonly used for data communications on IPS is Wireless Local Area Network (WLAN) and Bluetooth. Methods in determining applicable positions include triangulation, fingerprinting, and 
trilateration. The accuracy of the location determination depends on the environment of the reference point[2].

\section{B. Trilateration}

Trilateration is the process of calculating unknown point by using three points and distance from each point to unknown point [3]. The three points can be a circle or a triangle. In this study the three points are BLE beacons that become reference points. The formula is generated as follows:

$$
\begin{aligned}
& \text { Sphere A; } d_{a}{ }^{2}=\left(x-x_{a}\right)^{2}+\left(y-y_{a}\right)^{2} \\
& \text { Sphere B; } d_{b}{ }^{2}=\left(x-x_{b}\right)^{2}+\left(y-y_{b}\right)^{2} \\
& \text { Sphere C; } d_{c}{ }^{2}=\left(x-x_{c}\right)^{2}+\left(y-y_{c}\right)^{2}
\end{aligned}
$$

This formula is the basic formula of the Trilateration method so that it can be developed further to obtain better accuracy.

In calculating the distance to an unknown point, trilateration can use measurement of time of arrival (ToA) or distance based on received signal strength indication (RSSI). ToA is the time required by the signal from the mobile device to the refference point. In this case it is necessary to synchronize precisely between the transmitter and the receiver of the signal. RSSI is an indicator of signal strength between the transmitter and the signal receiver. Based on the value of RSSI we can estimate the distance between the signal receiver and the signal transmitter.

\section{Bluetooth Low Energy (BLE)}

Bluetooth is a wireless technology standard for exchanging data in close proximity to form a personal area network (PAN) with a high level of security. Bluetooth low energy also known as Bluetooth Smart is part of Bluetooth 4.0 Core Specification. The Bluetooth specification includes classic Bluetooth (a long-standing wireless standard for data exchange even today) and Bluetooth Low Energy (the newly introduced wireless standard on 4.0). Both these wireless connection standards cannot be connected to each other. There are several differences in the protocol layers in each of these two technologies that are barriers to interconnection.

Bluetooth Low Energy focuses on short-range communications. Actual distance from various wireless devices is influenced by many factors including place of operation, antenna design, tool orientation, and so on. The power of transmission (Tx power) is generally measured in units of $\mathrm{dBm}$ configured for a certain distance $(-30$ to 0 $\mathrm{dBm})$. The greater the transmission power will increase the range of the transmitted signal but the required power is greater so as to accelerate the usage life of the battery cell [4].

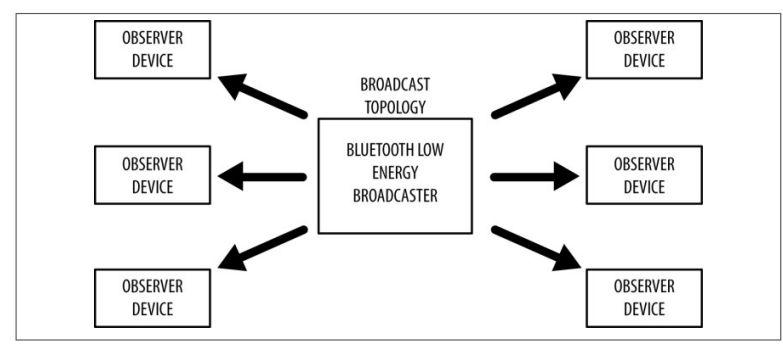

Figure 1. Broadcast Topology
In Fig. 1, this mechanism starts with a broadcaster that sends out data for all devices capable of receiving transmitted data. Broadcasting has two distinct roles: broadcaster and observer. Broadcasters work to send advertising packages periodically to everyone who wants to receive the package. The Observer performs a continuous search for receiving advertising packages that are being transmitted. Standard advertising package has a 31-byte length, the package is included with broadcaster data, but this package can also be made to contain specific information to be transmitted on other devices. BLE also provides an additional package or also called Scan Response that aims to request a second advertising frame with a 31byte length so a total of 62 bytes.

1) Cubeacon Card

Cubeacon Card is a BLE beacon that can be placed on various locations or objects. Cubeacon Card uses the iBeacon protocol with customizable parameters. Cubeacon also integrates with Mobile BackEnd as a Service (MbaaS) called Mesosphere and is useful in controlling and managing operational beacons. Cubeacon SDK is a library of Cubeacon for easy access between Android devices with Cubeacon Card. Cubeacon SDK has a function to determine the distance between beacon and another devices with the distance value in meter. Cubeacon card parameter can be seen in Table I.

TABLE I. CUBEACON CARD PARAMETER

\begin{tabular}{|l|l|}
\hline \multicolumn{1}{|c|}{ Item } & \multicolumn{1}{c|}{ Default Settings } \\
\hline UUID & $\begin{array}{l}\text { CB10023F-A318-3394-4199- } \\
\text { A8730C7C1AEC }\end{array}$ \\
\hline Major & 0 \\
\hline Minor & 0 \\
\hline Transmission Power & $6(0 \mathrm{dBm})$ \\
\hline Broadcast Interval & $900 \mathrm{~ms}$ \\
\hline
\end{tabular}

\section{SYSTEM DESIGN}

\section{A. UUID}

The new UUID design is useful for providing the information needed to determine the location of the device by the Trilateration method [5]. Such information is the floor and coordinates beacons (latitude and longitude). The UUID format is separated by commas to differentiate information so it is easy to process, for example: $30,-7 . x x x, 112 . x x x$. UUID format is divided into 3 parts, the first part contains floor information, floor information consists of 2digit number so that the second digit can be used for building which has half floor. The second part contains the latitude information of the Cubeacon Card and the third contains the longitude of the Cubeacon Card.

\section{B. Graph}

The process of locating the route on the system is using graph to store the required information. The nodes in the graph represent the point where the intersection occurs, the lines on the graph illustrate the direction that the user can 
pass to the next location, and the weight of the graph is the distance between nodes in meters.

\section{System Application}

System application on this research is divided into two systems, server systems and Android systems. The server system is a system for storing and receiving requests for data to be used by the Android system. The Android system is a system used by users to display in-building locations by utilizing Internet, Bluetooth and GPS features on Android devices. In addition, users can show another user's location by adding other users as friends. System design in general can be seen in Fig 2 .

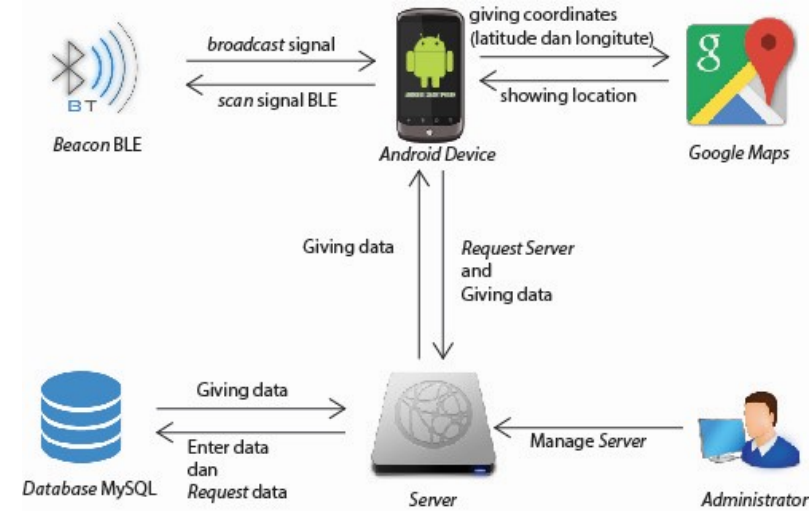

Figure 2. Design System

\section{SYSTEM RESULT}

\section{A. Beacon}

The testing is done using Cubeacon Card with $0 \mathrm{dBm}$ transmission power which is the maximum limit of transmission power that can be done, the greater the transmission power will increase the signal range from the beacon and also increase the beacon power consumption. Android device used is Sony Experia M4 Aqua (1.5GHz 4core processor, 2GB RAM). The test is done at Building $\mathrm{P}$ of the 3rd floor. Based on testing with various obstacles, Cubeacon Card position can affect the value of RSSI. As shown in Table 1, the test results can be concluded if the value of RSSI is closer to 0 , the better the signal. The optimal distance of Cubeacon Card signal that can be received by Android device is \pm 10 meters. This is influenced by environmental circumstances during testing such as WiFi signal interference. Testing result of Cubeacon Card can be seen in table II-III.

TABLE II. TESTING CUBEACON CARD Without OBSTACLE

\begin{tabular}{|c|l|c|}
\hline Obstacle & \multicolumn{1}{c|}{$\begin{array}{c}\text { Distances } \\
\text { (meter) }\end{array}$} & $\begin{array}{c}\text { RSSI } \\
\text { (dBm) }\end{array}$ \\
\hline \multirow{4}{*}{ No obstacle (line of sight) } & 1 & -63 \\
\cline { 2 - 3 } & 2 & -66 \\
\cline { 2 - 3 } & 3 & -71 \\
\cline { 2 - 3 } & 4 & -71 \\
\cline { 2 - 3 } & 5 & -74 \\
\cline { 2 - 3 } & 6 & -79 \\
\hline
\end{tabular}

\begin{tabular}{|l|l|l|}
7 & -80 \\
\cline { 2 - 3 } & 8 & -79 \\
\cline { 2 - 3 } & 9 & -80 \\
\cline { 2 - 3 } & 10 & -80 \\
\hline
\end{tabular}

TABLE III. TESTING CUBEACON CARD WITH OBSTACLE WOODEN DOOR

\begin{tabular}{|l|l|l|}
\hline Obstacle & $\begin{array}{c}\text { Distances } \\
\text { (meter) }\end{array}$ & $\begin{array}{c}\text { RSSI } \\
\text { (dBm) }\end{array}$ \\
\hline \multirow{5}{*}{$\begin{array}{l}\text { Wooden } \\
\text { door }\end{array}$} & 1 & -78 \\
\cline { 2 - 3 } & 2 & -73 \\
\cline { 2 - 3 } & 3 & -77 \\
\cline { 2 - 3 } & 4 & -81 \\
\cline { 2 - 3 } & 5 & -82 \\
\cline { 2 - 3 } & 6 & -89 \\
\cline { 2 - 3 } & 7 & -91 \\
\cline { 2 - 3 } & 8 & -87 \\
\cline { 2 - 3 } & 9 & -88 \\
\cline { 2 - 3 } & 10 & -90 \\
\hline
\end{tabular}

\section{1) Calibration}

Calibration on beacons is useful for adjusting RSSI values at a distance of 1 meter so that the estimated distance from the beacon to the device more accurate. Calibration can be done by using Cubeacon Tools app on Android device. Results of RSSI value at a distance of 1 meter by performing calibration vary widely. This is influenced by the interference that occur around the Cubeacon Card and Android devices. Calibration does not always work,needs to be done several times to succeed. It is also influenced by other factor such as WiFi signal. Calibration notification can be seen in Fig. 3.

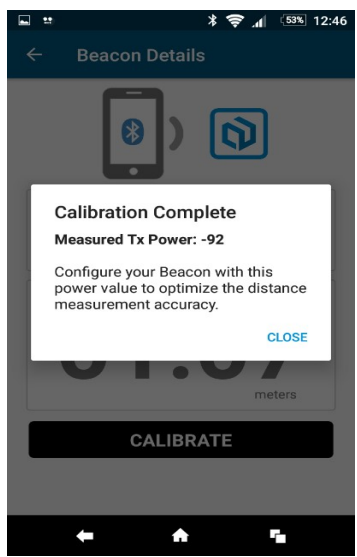

Figure 3. Calibration notification

\section{B. Trilateration Calculation}

Test results based on table IV, latitude and longitude coordinates do not produce the expected value. So we use Trilateration library to calculate the location of the device.

TABLE IV. TRILATERATION CALCULATION 


\begin{tabular}{|c|l|l|c|r|}
\hline \multicolumn{9}{|c|}{ Beacon } & latitude & longitude \\
\hline \multirow{2}{*}{1} & $\mathrm{x}$ & -7.339443145697 & -1869901762 & 145139307.5 \\
\cline { 2 - 3 } & $\mathrm{y}$ & 112.73854997009 & & \\
\cline { 2 - 3 } & $\mathrm{z}$ & 0.7729623 & & \\
\hline 2 & $\mathrm{x}$ & -7.339367994109 & & \\
\cline { 2 - 3 } & $\mathrm{y}$ & 112.738486938178 & & \\
\cline { 2 - 3 } & $\mathrm{z}$ & 0.8428684 & & \\
\hline 3 & $\mathrm{x}$ & -7.339493690120 & & \\
\cline { 2 - 3 } & $\mathrm{y}$ & 112.738441675901 & & \\
\cline { 2 - 3 } & $\mathrm{z}$ & 1.0805551 & & \\
\hline
\end{tabular}

\section{Android Application}

Mobile apps that have been made will calculate the location on a digital map that is described as a marker on the direction page as shown in Fig. 4.

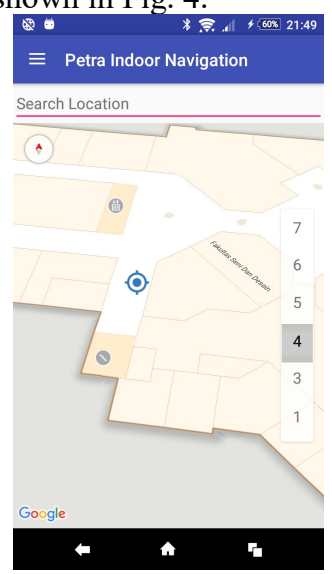

Figure 4. Direction Page

The user can also display another user's location on the map by adding another user as a friend as shown in Fig. 5 and Fig. 6. The friends that show up on the map will update every 5 seconds as seen in Fig. 7.

The direction page displays a digital map using the Google Maps Android API. In addition, this page displays the user's position and provides a route to the destination location. The device does a ranging on the background process to determine the location of the device that requires 3 nearest beacon signals to the device, then the application sends the Cubeacon Card information in the Mesosphere cloud to get the beacon location information because Cubeacon Tools does not allow UUID parameters on the Cubeacon Card to be changed. In this test used Cubeacon Android SDK to get information from Cubeacon Card through function already available.

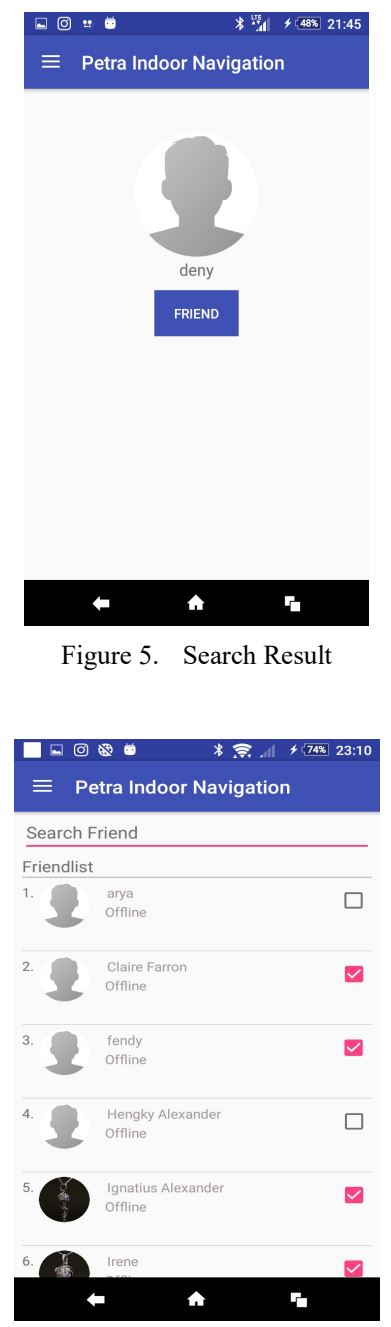

Figure 6. Friendlist

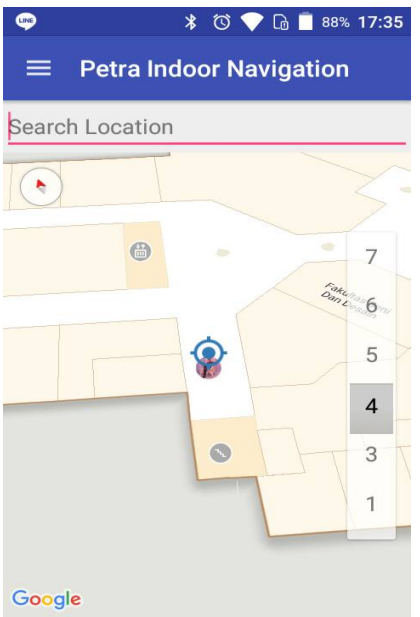

Figure 7. Direction page (showing other user) 
This test is done to see the accuracy of the location of the user to the digital map based on the upload and download speed of the device with the condition of the user running in the area around the beacon. The internet connection used is packet data of Telkomsel and XL providers on HSPA + (High Speed Packet Access) and LTE (Long Term Evolution) network connections. Accuracy testing is performed with the device being in the same position for 5 minutes. During the test location error occurred 16 times with total displacement of 100 times, so the accuracy of determining the location is about $84 \%$.

\section{CONCLUSION}

The conclusion of this research are:

- The digital map using Google Maps Javascript API version 3.29.1 does not support building level features, this feature can be replaced with custom overlay provided by Google Maps Javascript API.

- The calibration process on the Cubeacon Card results in inconsistent values and the optimal distance of Cubeacon Card against the device to detect signals up to \pm 10 meters.

- The accuracy of the user location is influenced by the distance estimation of the beacons, the number of signals captured by Android devices, and the internet connection from the Android device with an accuracy of $84 \%$.

- The formula determines the location of the device based on the journal cited the author does not produce the expected value and Trilateration method by using metadata stored in the cloud does not give the expected results because the location determination process depends on the internet connection and cloud availability.

- Application performance is influenced by the stability and speed of internet connection especially the direction feature.

- This application can provide a route to the destination by using Dijkstra's data graph and library to determine the closest route.

\section{ACKNOWLEDGMENT}

This research uses cubeacon device grant from PT. Eyro Digital Technology. Thank you for the help and cooperation between Faculty of Industrial Technology Petra Christian University with PT. Eyro Digital Technology.

\section{REFERENCES}

[1] A. Blattner., Y. Vasilev, \& B.H. Mühlbauer. Mobile Indoor Navigation Assistance for Mobility Impaired People. 6th International Conference on Applied Human Factors and Ergonomics (AHFE 2015) and the Affiliated Conferences (p. 8). Las Vegas: Elsevier B.V. 2015.

[2] R. Chen. Ubiquitous Positioning and Mobile Location-Based Services in Smart. Hershey: Information Science Reference. 2012.

[3] O. Samuel, E. Herbert, \& O. Anthony. Trilateration Based localization Algorithm for Wireless Sensor Network. International Journal of Science and Modern Engineering. 2013.

[4] K. Townsend, C. Cufi, Akiba, \& R. Davidson. Getting Started with Bluetooth Low Energy. Sebastopol: O'Reilly Media, Inc. 2014.

[5] V. Varshney, R. K. Goel, \& M. Qadeer. A.Indoor Positioning System Using Wi-Fi \& Bluetooth Low Energy Technology. 2016 Thirteenth International Conference on Wireless and Optical Communications Networks (WOCN) (pp. 1-6). Hyderabad: Institute of Electrical and Electronics Engineers. 2016. 